\title{
KRN7000 结构类似物及其免疫活性研究进展
}

\author{
冯俊娜 ${ }^{a, b}$ 高 芳 ${ }^{a}$ 彭绍辉 $b$ 陈华*,a 李小六*, $a$ \\ ( ${ }^{a}$ 河北大学化学与环境科学学院 保定 071002) \\ $\left({ }^{b}\right.$ 中国地质大学长城学院 保定 071000)
}

\begin{abstract}
摘要 KRN7000 具有广泛的生物活性，如抗肿瘤、抗结核病、抗真菌、抗病毒、消炎以及治疗自身免疫性疾病等. 作 为免疫调节剂, 其免疫作用主要通过活化自然杀伤 $\mathrm{T}$ 细胞(NKT), 促进细胞因子 IFN- $\gamma$ 和 IL-4 的释放. 由于 IFN- $\gamma$ 抑制 $\mathrm{T}_{\mathrm{H}} 2$ 活性, IL-4 抑制 $\mathrm{T}_{\mathrm{H}} 1$ 活性, $\mathrm{T}_{\mathrm{H}} 1$ 和 $\mathrm{T}_{\mathrm{H}} 2$ 生物学效应的相互抑制限制了 KRN7000 在临床治疗上的应用. 因此，对 KRN7000 的结构进行修饰, 以期得到免疫作用更好的药物是近年来研究的热点. 就 KRN7000 最新结构改造及免疫活 性研究进展进行综述.
\end{abstract}

关键词 KRN7000; 免疫调节剂; $\mathrm{T}_{\mathrm{H}} 1 / \mathrm{T}_{\mathrm{H}} 2$

\section{Research Advances of KRN7000 Analogues and Their Immune Activities}

\author{
Feng, Junna ${\text { Gao, } \text { Fang }^{a} \quad \text { Peng, Shaohui }}^{b} \quad$ Chen, Hua*,a $^{*} \quad$ Li, Xiaoliu*,a \\ ( ${ }^{a}$ College of Chemistry and Environmental Science, Hebei University, Baoding 071002) \\ ( ${ }^{b}$ China University of Geosciences Great Wall College, Baoding 071000)
}

\begin{abstract}
KRN7000 has widely biological activities, such as anti-tumor, anti-tuberculosis, anti-fungal, anti-virus, anti-inflammation, and against auto-immune diseases. As the potential immunomodulator, KRN7000 can effectively activate the natural killer T cells (NKT cells) and induce the secretion of IFN- $\gamma$ and IL-4 cytokines. However, IFN- $\gamma$ inhibited the activity of $\mathrm{T}$ helper 2 cells $\left(\mathrm{T}_{\mathrm{H}} 2\right)$ and IL-4 inhibited the activity of $\mathrm{T}$ helper 1 cells $\left(\mathrm{T}_{\mathrm{H}} 1\right)$, which mediated humoral as well as celluar immune reactions, respectively. The concomitant of functions of $T_{H} 1$ and $T_{H} 2$ limits the therapeutic potential of KRN7000 against immune diseases. Therefore, developing new analogues of KRN7000 to find new immnostimulating drugs has drawn many attentions in the world. This review presents the recent progress in the study on the structure and immune activity of the synthesized KRN7000 derivatives.
\end{abstract}

Keywords KRN7000; immunomodulator; $\mathrm{T}_{\mathrm{H}} 1 / \mathrm{T}_{\mathrm{H}} 2$

KRN7000 (1, 图 1)是 1994 年 Natori 等 ${ }^{[1]}$ 从日本冲 绳附近海域的海绵组织中分离得到的 $\alpha$-糖苷键半乳糖 神经酰胺 ( $\alpha$-GalCer). 它具有广泛的生物活性, 如抗肿 瘤 $^{[2] 、 \text { 抗结核病 }{ }^{[3]} \text { 、抗病毒 }}{ }^{[4]}$ 、抗真菌 ${ }^{[5]}$ 、消炎 ${ }^{[6]}$ 以及治 疗自身免疫性疾病(如系统性红斑狼疮 ${ }^{[7]}$ 、糖尿病 $\left.{ }^{[8]}\right)^{\text {等. }}$ 其中, 其高效的免疫调节活性最引人注目. 研究表明 KRN7000 可以通过活化自然杀伤 $\mathrm{T}$ 细胞(NKT)促进细 胞因子 IFN- $\gamma$ 和 IL-4 的释放 ${ }^{[9]}$. KRN7000 可与 CD1d 蛋
白(CD1 蛋白质的一种, 可通过分泌脂质抗原活化 NKT 细胞)识别结合, 形成 CD1d/KRN7000 二元复合物, 进 而被 NKT 细胞表面的受体(TCR)识别, 形成具有免疫活 性的 “CD1d 蛋白/KRN7000/NKT 细胞”三元复合物, 交互刺激 $\gamma$ 干扰素(IFN- $\gamma$ )及白介素 4 (IL-4)两大细胞因 子的释放 ${ }^{[10,11]}$. 这些细胞因子分别诱导 $\mathrm{T}$ 辅助细胞 1 $\left(\mathrm{T}_{\mathrm{H}} 1\right.$, 介导细胞免疫) 和 $\mathrm{T}$ 辅助细胞 $2\left(\mathrm{~T}_{\mathrm{H}} 2\right.$, 介导体液免 疫)的分化. $\mathrm{T}_{\mathrm{H}} 1 / \mathrm{T}_{\mathrm{H}} 2$ 在体内处于动态平衡, 保证人体的

\footnotetext{
* E-mail: lixl@hbu.cn and hua-todd@163.com; Tel. \& Fax.: 0086-312-5971116

Received September 30, 2014; revised December 6, 2014; published online December 18, 2014.

Project supported by the National Natural Science Foundation of China (Nos. 20972039, 21372060), the Research Fund for the Doctoral Program of Higher Education of China (No. 20121301110004), the Medicinal Joint Funds of the Natural Science Foundation of Hebei and Shijiazhuang Pharmaceutical Group Foundation (No. B2011201169), and the Natural Science Foundations of Education Department of Hebei Province (No. ZH2011110).

国家自然科学基金(Nos. 20972039, 21372060)、教育部博士点基金(No. 20121301110004)、河北省自然科学基金石药集团医药联合基金(No. B2011201169)、河北省教育厅自然科学基金(No. ZH2011110)资助项目.
} 
免疫系统处于平衡状态. 由于 IFN- $\gamma$ 抑制 $\mathrm{T}_{\mathrm{H}} 2$ 活性, IL-4 抑制 $\mathrm{T}_{\mathrm{H}} 1$ 活性, $\mathrm{T}_{\mathrm{H}} 1$ 和 $\mathrm{T}_{\mathrm{H}} 2$ 生物学效应的相互抑制限制 了 KRN7000 在临床治疗上的应用. 因此, 对 KRN7000 进行结构修饰, 以实现 NKT 细胞选择性的分化 $\mathrm{T}_{\mathrm{H}} 1$ 或 $\mathrm{T}_{\mathrm{H}} 2$ 细胞, 发挥其更好的免疫作用, 成为该类化合物的 研究热点 ${ }^{[12 \sim 15]}$.

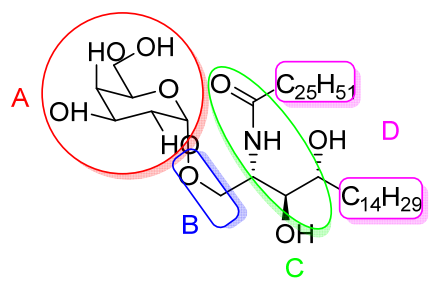

图 1 KRN7000 的结构式

Figure 1 The structure of KRN 7000

\section{KRN7000 的免疫作用机理研究}

从结构上看, KRN7000 由糖环 $(\mathrm{A}) 、$ 糖苷键(B)、神 经酰胺 (C) 和脂肪链 (D) 四部分组成 (图 1). 其中, KRN7000 的两个长脂链可以分别进入 CD1d 蛋白 $N$ 端 的两个非极性口袋, 实现与 CD1d 蛋白的稳定结合, 而 糖基嵌插在蛋白的螺旋结构中. X 射线结构分析证明氢 键对结构的稳定性起到重要作用(图 2). CD1d 蛋白的 Asp151 氨基酸与糖基的 2-OH 及 3-OH 之间、 $\mathrm{Thr} 154$ 与 Asp151 以及酰胺基之间都存在氢键作用, 称为 OTAN 氢键网 ${ }^{[13]}$. 而 $\operatorname{Trp} 153$ 与糖基间的范德华力可提高三元 复合物的稳定性.

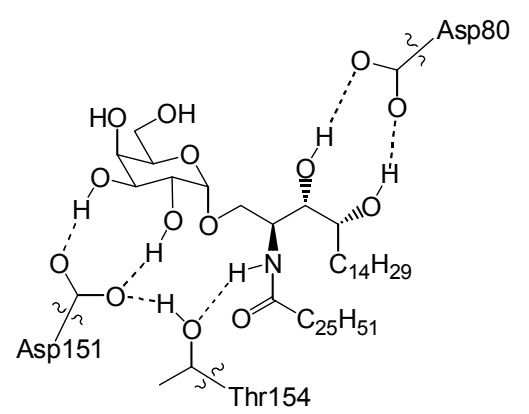

图 2 KRN7000 与 CD1d 蛋白之间的氢键体系

Figure 2 Hydrogen bond network in the binary complex of KRN7000 and CD1d

根据 Nadas 等 ${ }^{[16]}$ 对三元复合物结合位点的计算, CD1d 氨基酸残基 Glu83、Arg95 和 Asp80 在与 TCR 识 别中起主导作用. 当 $\mathrm{CD} 1 \mathrm{~d} / \mathrm{KRN} 7000 / \mathrm{TCR}$ 三者有效结 合后, NKT 细胞就会选择性释放 IFN- $\gamma$ 和 IL-4(分别为 $\mathrm{T}_{\mathrm{H}} 1$ 和 $\mathrm{T}_{\mathrm{H}} 2$ 免疫的特征因子). 其中, 二元复合物 (CD1d/KRN7000)的稳定性对细胞因子的释放起重要作 用. 当 KRN7000 和 CD1d 结合力较差(易分解)时, 将导 致 NKT 刺激的半衰期缩短. 因此, 提高复合物的稳定
性, 选择性地使 $\mathrm{NKT}$ 细胞分化 $\mathrm{T}_{\mathrm{H}} 1$ 或 $\mathrm{T}_{\mathrm{H}} 2$ 细胞, 成为 KRN7000 结构改造的重要方向.

\section{KRN7000 的结构改造}

\section{1 糖环 $\mathrm{A}$ 的结构修饰}

\subsection{1 糖基修饰}

Kawano 等 ${ }^{[17]}$ 分别用葡萄糖、甘露糖代替 KRN7000 中的半乳糖基(图 3), 构效关系(SAR)分析表明 $\alpha$ - $D$-半乳 糖神经酰胺的活性比 $\alpha-D$-葡萄糖神经酰胺(2)的活性好, 而后者的活性高于 $\alpha-D$-甘露糖神经酰胺(3)的活性. 此 结果和 CD1d 与各种单糖结合能力的强弱是一致的, 而 $\alpha-D$-甘露糖修饰的类似物可以优先被 $\mathrm{CD} 1 \mathrm{~b}$ (其他 $\mathrm{CD} 1$ 蛋白质)识别.
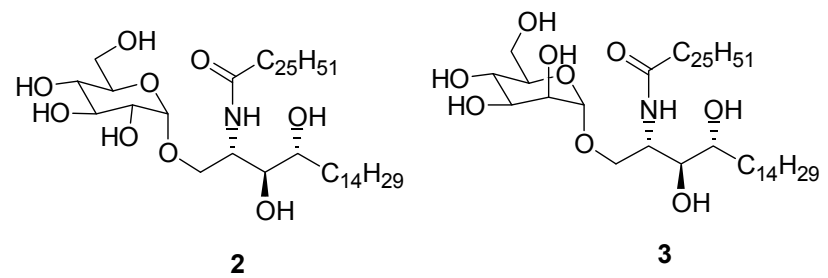

图 3 糖基取代的 KRN7000 类似物

Figure 3 Sugar substituted KRN7000 analogs

通过对糖环 2, 3, 4 位羟基进行氟原子 $(\mathbf{4} \sim \mathbf{6})^{[18] \text { 、甲 }}$ 氧基(7)、磺酸基 $(8)^{[19]}$ 取代及羟基脱除 $(9 、 10)$ 验证了羟 基对活性的重要性(表 1). 结果显示: 2 位羟基是最重要 的，用其它基团取代(如 4、7)会使化合物的活性显著降 低或丧失; 3、4 位羟基的重要性次于 2 位, 氟原子取代 的 5 和 6 以及羟基脱除的 9 和 10 依然可以释放 IL-2 细 胞因子; 3 位羟基被磺酸基取代的化合物 $\mathbf{8}$, 同等浓度下 其活性与 KRN7000 相当, 磺酸基的引入使化合物保持 了氢键网的完整性, 从而与 TCR 结合更好. 分子对接模 型表明 4 位羟基可以被其他基团如脂肪胺、酯或者醚类 基团取代, 方向和氢键均没有改变, 而 $2 、 3$ 位酰胺链修 饰得到的类似物与 $\mathrm{CD} 1 \mathrm{~d}$ 结合较差 ${ }^{[20]}$. 此外, 当 $\alpha$ - $D$-半 乳糖的 $2 、 3$ 位(11、12)或 6 位(13)连上其他的糖基时 ${ }^{[20,21]}$, 活性较 KRN7000 并没有降低, 可能是因为糖基水解后 仍可得到 KRN7000, 而使活性损失小.

相对而言, 由于 6 位羟基在活性接触位点之外, 因 此 6 位着基对活性的影响最小, 该位羟基修饰后的化合 物(14 19, 表 2)均有较高活性. 虽然这些化合物大多数 没有表现出更好的刺激 NKT 细胞活化的作用, 但化合 物 $14 \sim 16^{[22]}$ 却产生了一定的 $\mathrm{T}_{\mathrm{H}} 1$ 极化, 这种现象可能与 CD1d 蛋白上的 Trp153 和 6 位基团的相互作用有关. $\operatorname{Trp} 153$ 中富电子的吲哚可以和化合物中的芳环发生 $\pi$ 电子堆积作用, 有利于加强复合物稳定性. 化合物 $17^{[23,24]}$ 的活性显著增加：一方面，甲氧基的给电子效应 
表 1 糖环 2, 3, 4 位羟基改造的 KRN7000 类似物

Table 1 Substitution in positions 2, 3 or 4 of the sugar in KRN7000

\begin{tabular}{|c|c|c|c|c|c|}
\hline Analog & $\mathrm{R}^{1}$ & $\mathrm{R}^{2}$ & $\mathrm{R}^{3}$ & $\mathrm{R}^{4}$ & Activity \\
\hline 4 & $\mathrm{~F}$ & $\mathrm{OH}$ & $\mathrm{OH}$ & $\mathrm{OH}$ & \\
\hline $5^{b}$ & $\mathrm{OH}$ & $\mathrm{F}$ & $\mathrm{OH}$ & $\mathrm{OH}$ & Weak $^{c}$ \\
\hline $6^{b}$ & $\mathrm{OH}$ & $\mathrm{OH}$ & $\mathrm{F}$ & $\mathrm{OH}$ & Weak \\
\hline 7 & $\mathrm{OMe}$ & $\mathrm{OH}$ & $\mathrm{OH}$ & $\mathrm{OH}$ & \\
\hline 8 & $\mathrm{OH}$ & $\mathrm{OSO}_{3} \mathrm{Na}$ & $\mathrm{OH}$ & $\mathrm{OH}$ & Weak \\
\hline 9 & $\mathrm{OH}$ & $\mathrm{H}$ & $\mathrm{OH}$ & $\mathrm{OH}$ & Weak \\
\hline 10 & $\mathrm{OH}$ & $\mathrm{OH}$ & $\mathrm{H}$ & $\mathrm{OH}$ & Weak \\
\hline 11 & $\alpha$-Galactopyranose & $\mathrm{OH}$ & $\mathrm{OH}$ & $\mathrm{OH}$ & Similar ${ }^{d, e, f}$ \\
\hline 12 & $\mathrm{OH}$ & $\beta$-Galactofuranose & $\mathrm{OH}$ & $\mathrm{OH}$ & Similar ${ }^{d, e}$ \\
\hline 13 & $\mathrm{OH}$ & $\mathrm{OH}$ & $\mathrm{OH}$ & $\alpha$-Galactopyranose & Similar $^{d}$ \\
\hline
\end{tabular}

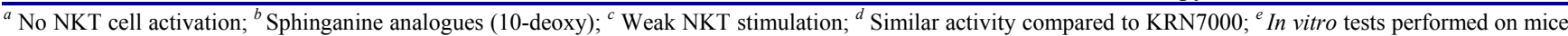
cells; ${ }^{f}$ In vitro tests performed on human cells.

表 2 对糖基 6 位羟基修饰的 KRN7000 类似物

Table 2 Substitution in positions 6 of the sugar

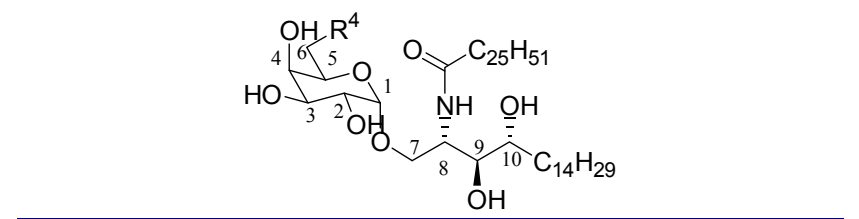

\begin{tabular}{lll}
\hline Analog & $\mathrm{R}^{4}$ & Activity $^{a}$ \\
\hline
\end{tabular}

14

$\begin{array}{ll}\mathbf{1 5} & \text { NH-1-naphthalenyl } \\ \mathbf{1 6} & \text { Substitued 1,2,3-triazole } \\ \mathbf{1 7} & \text { OMe } \\ \mathbf{1 8} & \text { COOH } \\ \mathbf{1 9} & \text { NHCO-PEG-Me }\end{array}$<smiles>CNC(=O)c1ccc(Cl)c(C(F)(F)F)c1</smiles>

$\mathrm{T}_{\mathrm{H}} 1^{b, c}$

$\mathrm{T}_{\mathrm{H}} 1^{b, d}$

$\mathrm{T}_{\mathrm{H}} 1^{c, e}$

$\mathrm{T}_{\mathrm{H}} 1^{b, c}$

$\mathrm{T}_{\mathrm{H}} 2^{c, f}$
${ }^{a}$ NKT stimulation activity compared to KRN7000; ${ }^{b}$ Slight increasing of $\mathrm{T}_{\mathrm{H}} 1$ stimulating activity compared to KRN7000. ${ }^{c}$ In vitro tests performed on human cells. ${ }^{d}$ In vivo/intro tests performed on mice cells. ${ }^{e}$ Marked increasing of $\mathrm{T}_{\mathrm{H}} 1$ stimulating activity compared to KRN7000. ${ }^{f}$ Slight increasing of $\mathrm{T}_{\mathrm{H}} 2$ stimulating activity compared to KRN7000.

使 4 位羟基的电子云密度增加，且 $4 、 6$ 位羟基间的氢键 消失，导致 4 位羟基更易被 TCR 识别; 另一方面，甲基 的存在导致化合物和 Trp153 有更强的范德华力, 加强 三元复合物的整体稳定性; 最后, 细胞因子 IL-12 的大 量产生促进了 IFN- $\gamma$ 的增加和 IL-4 的减少. 以上三方面 共同导致了 $\mathrm{T}_{\mathrm{H}} 1$ 细胞的显著增加. 化合物 $\mathbf{1 8}^{[25]}$ 诱导 $\mathrm{T}_{\mathrm{H}} 1$ 分化的原因与复合物的稳定性增加以及 IL-12 的轻微增
加有关 ${ }^{[26]}$. 以上发现为设计合成新的潜在的 $\mathrm{T}_{\mathrm{H}} 1$ 极化的 免疫药物提供了理论依据. 为了增加化合物的水溶性, 通过酰胺键在糖的 6 位连接上甲基-PEG 基团(19) ${ }^{[27]}$. 该 水溶物保留了对 CD1d 受体的特异性结合和对免疫细胞 的刺激作用, 表现出较好的生物学性能, 它是第一个诱 导 $\mathrm{T}_{\mathrm{H}} 2$ 类型免疫反应的类似物.

\subsection{2 多羟基醇修饰}

以六元环醇基代替半乳糖，所得结构类似物 $20 \sim$ 23(图 4)可以显著增加 IFN- $\gamma$ 的释放而抑制 IL-4 的释放, 对 $\mathrm{T}_{\mathrm{H}} 1$ 有极强的诱导作用 ${ }^{[28]}$. 而肌醇基取代类似物 $\mathbf{2 4} \sim$ 27 的活性却较 KRN7000 的差. 直链式多元醇取代的类 似物 28 虽然是非糖基结构, 但分子可以较灵活的与 TCR 的氨基酸残基 Phe29、Ser30 和 Gly96 形成多个稳 定的氢键, 其对人和鼠的 NKT 细胞对比 KRN7000 均有 更好的活化作用 ${ }^{[29,30]}$.

\section{2 糖苷键 B 的结构修饰}

\section{$2.2 .1 C$-糖苷}

相对而言, $C$-糖苷由于碳碳键的存在较其他糖苷的 半缩醛结构, 稳定性大大提高. 为了增加 KRN7000 的 稳定性, $C$-糖苷类似物(图 5)成为不错的选择. 2002 年, Kotobuki 制药公司 ${ }^{[31]}$ 第一次对 $\alpha-C-K R N$ (29)进行了活 性测试. 和 KRN7000 相比, $\alpha-C-\mathrm{KRN}$ 表现出对黑瘤素 B16 较好的 $\mathrm{T}_{\mathrm{H}} 1$ 诱导效应. 最近研究表明, 微克级的 29 可以有效抑制鼠肺癌细胞转移, 其效果约是 KRN7000 的百倍, 而在治疗鼠疮疾的效果是 KRN7000 的 1000 倍, 


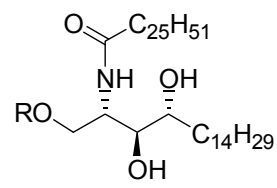

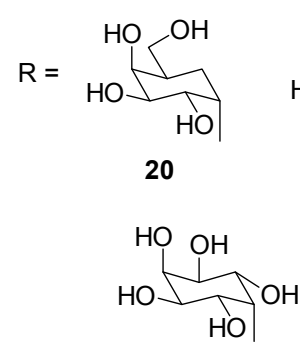

25<smiles>CC1(O)CCC(O)C(O)C1O</smiles>

21

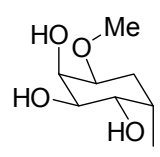

22<smiles>OC1CCCC2(O)CCCC1C2O</smiles>

23<smiles>[Z10]C1C(O)C(O)C(O)C(O)C1(O)CO</smiles>

24

图 4 多羟基醇修饰的类似物

Figure 4 Polyhydroxy-alcohol modified analogs

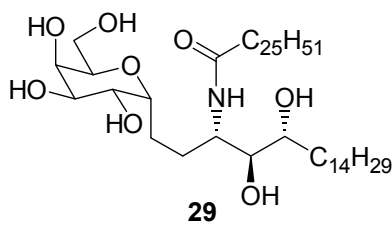

29

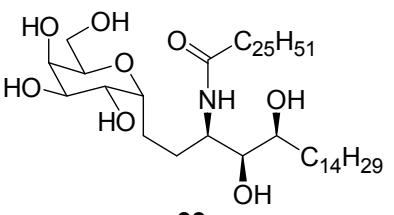

33

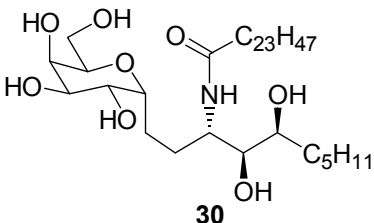

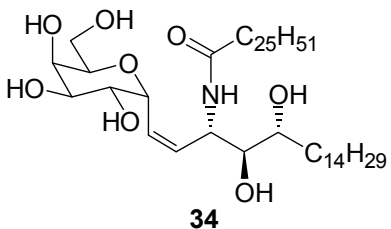

34

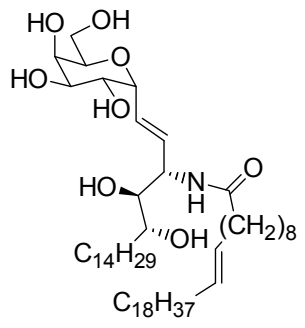

38

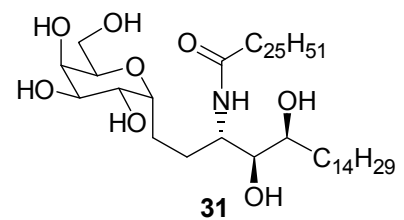

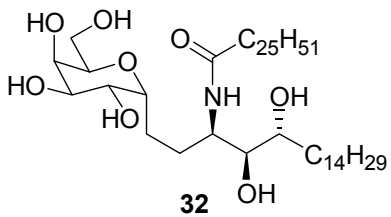

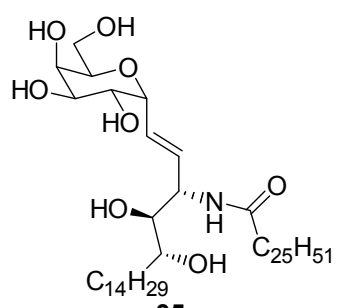
35

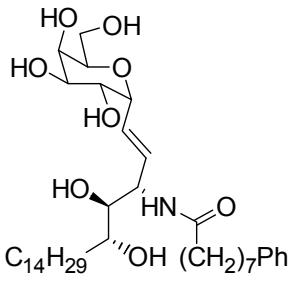

36

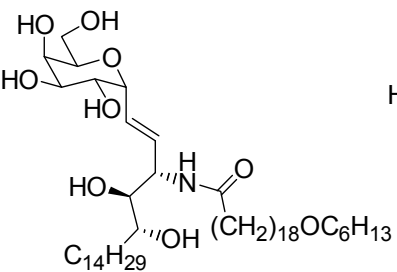

39<smiles>CCCCCCCCCCCCCCCCCCC(O)C(O)C(O)C(O)CO</smiles>

40

图 $5 \mathrm{KRN7000}$ 的 $C$-糖苷类似物

Figure $5 C$-glycoside analogs of KRN7000

达到纳克级 ${ }^{[32]}$. 虽然人和鼠的CD1d都可以和 29 很好的 结合, 但是人的NKT细胞并不能有效识别相应的二元复 合物, 其原因可能是人和鼠的NKT细胞在结构上存在差 别.

由于 $\alpha-C-\mathrm{KRN}$ (29)显著的活性增加，使得其合成、 结构改造及活性研究成为热点 ${ }^{[33 \sim 35]}$. Toba等合成了烷基 链缩短的 $C-\mathrm{KRN}-\mathrm{OCH}(\mathbf{3 0})^{[36]}$ 以及 $F r a n c k$ 等合成了 $9 、 10$ 位羟基构型不同的非对映异构体 $31 \sim \mathbf{3 3}^{[37]}$. 类似物的 活性虽然均较29的差, 但值得注意的是, 30虽然不能诱 导小鼠体外脾细胞IL-4和IFN- $\gamma$ 的产生, 但却可以有效 增加小鼠体内血清细胞IL-4的浓度. 但值得注意的是, Franck 等 ${ }^{[13,38]}$ 合成了糖基和神经酰胺之间由双键连接 的化合物 34 和 35 , 其中, $E$ 式 $(35)$ 对 $\mathrm{T}_{\mathrm{H}} 1$ 细胞的诱导作用
比KRN7000好，但弱于化合物 29; $Z$ 式(34)则几乎没有活 性。在此基础上, 进一步合成了化合物 36 39. 相对于 化合物 29, 在酰胺处连接缩短的脂肪链末端引入芳香环 的类似物 36 以及含烯烃链的 37 和 38 都能显著诱导 $\mathrm{T}_{\mathrm{H}} 1$ 分 化, 而引入醚链的39则没有表现出对 $\mathrm{T}_{\mathrm{H}} 1$ 的诱导作用. 有趣的是, 虽然 36 和 37 均能有效地激活活化人的NKT细 胞，但在小鼠实验中，37表现出体外测试活性，而36在 小鼠体内和体外均没有活性 ${ }^{[39]}$.

为了验证短连接链对活性的影响, Bittman 等 ${ }^{[40]}$ 合 成了 $C$-糖苷类似物 40 . 体外实验结果表明, 虽然 40 对 NKT 的活化作用不如 $\mathbf{2 9}$, 但却可以大幅度的提高 IFN- $\gamma / \mathrm{IL}-4$ 和 IFN- $\gamma / \mathrm{IL}-13$ 的比例, 可作为典型的 $\mathrm{T}_{\mathrm{H}} 1$ 型 免疫增强剂. 


\subsubsection{S-糖苷及其它}

因为 $S$-糖苷衍生物的化学结构和分解代谢能力都 很稳定, 所以普遍认为化合物 41(图 6)的活性在体内应 能持续较长时间 ${ }^{[41]}$. 但研究表明, 41 无论是在体内还是 体外都没有活性, 由于 $\mathrm{S}-\mathrm{C}$ 键 $(182 \mathrm{pm})$ 比 $\mathrm{O}-\mathrm{C}$ 键 $(143$ $\mathrm{pm})$ 长, 化合物中的两个 $\mathrm{S}-\mathrm{C}$ 键可能导致与对应的识 别位点的错位, 进而引起活性丧失. 此外, 在半乳糖苷 和神经酰胺中间增加 4 个原子 $(42)^{[42]}$, 或者是以 $\alpha$ 和 $\beta$ 两种构型肜连接的化合物 $\mathbf{4 3}$ 和 $\mathbf{4 4}{ }^{[43]}$, 均没有活性, 连 接臂上多出的 $\mathrm{N}$ 原子可能对活性不利. 由此可见, 糖基 和神经酰胺之间的连接链延长, 将对化合物的活性有大 的负面影响. 最近, Murphy 等 ${ }^{[44]}$ 利用 Click 反应合成了 三唑连接的类似物 45 , 但未见活性报道.

\section{3 神经酰胺(C)的结构修饰}

\subsection{1 鞘氨醇的结构改造}

化合物 $46 \sim \mathbf{5 0}^{[45]}$ (图 7) 是 8、9、10 位构型改变的 KRN7000 类似物. 构效关系分析表明, 8 位酰胺的立体 构型对活性影响最大, 9 位羟基其次, 而 10 位羟基的构 型改变对活性影响不大. 9、10 位羟基对 TCR 的识别有 既独立又协同的重要作用. 同时去除 9、10 位羟基(51) 将导致活性消失, 而只去除 10 位羟基或 9 位羟基的化合 物 $\mathbf{5 2}^{[46]}$ 和 $\mathbf{5 3}^{[47]}$ 对 NKT 细胞的刺激作用均和 KRN7000 相当, 但其较差的溶解度可能会成为它作为潜在药物的 限制因素. 将 9 位羟基用一个(54)或者两个氟原子(55) 取代, 可以得到极化 $\mathrm{T}_{\mathrm{H}} 1$ 的衍生物 ${ }^{[48]}$, 可能的原因是氟 原子的强吸电子效应可以加强与 $\mathrm{CD} 1 \mathrm{~d}$ 氨基酸残基 Asp80 的氢键结合, 从而增加二元复合物的稳定性. 如

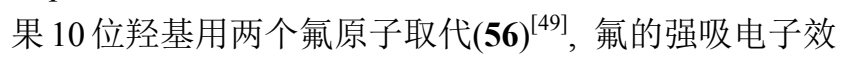
应也将导致 9 位羟基与 Asp80 的氢键作用增加, 致使化 合物对 $\mathrm{T}_{\mathrm{H}} 1$ 的刺激作用增强. 而 9 位或 10 位的羟基用

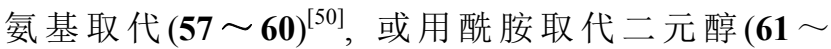

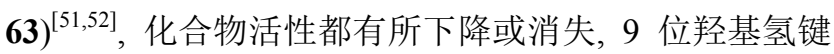
作用的减弱或消失, 可能是此类化合物活性降低的主要 原因.

为了增加亲水性, Shiozaki 等 ${ }^{[53,54]}$ 合成了 9 个多羟 基取代鞘氨醇链的 KRN7000 类似物(64 72)(图 8). 其 中, 64 和 66 释放细胞因子 IFN- $\gamma$ 的能力强于 KRN7000, 因此更倾向于诱导 $\mathrm{T}_{\mathrm{H}} 1$ 的分化; 而 65 和 68 的活性稍弱 于 KRN7000; 67 的活性则与 KRN7000 相当. 69 72 促 进 IL-4 的释放能力远远强于其对 IFN- $\gamma$ 的刺激, 因此, 这三种多羟基类似物是 $\mathrm{T}_{\mathrm{H}} 2$ 型免疫增强剂, 同时表明, 酰胺侧链上含有 $2 \sim 3$ 个羟基将对 NKT 细胞选择性的分 化 $\mathrm{T}_{\mathrm{H}} 2$ 起重要作用. 此外, 69、70 和 72 对脑脊髓炎也有 很好的抑制作用. Sawant 等 ${ }^{[55]}$ 通过不同的糖开环策略, 立体选择性地合成了鞘氨醇多羟基类似物 64 及其 9 位 和 11 位的非对映异构体 73 75, 这些鞘氨三醇衍生物 均能有效刺激小鼠淋巴瘤细胞分泌白细胞介素-2 (IL-2), 且与 KRN7000 活性相当.

\subsection{2 酰胺的结构改造}

用磺酰胺取代酰胺的化合物 76(图 9) 明显促进 IL-4 的释放, 而用酰胺的等电子体三氮唑取代酰胺键得到的 类似物 77, 其释放 IL-4 的能力强于释放 IFN- $\gamma^{[56]}$, 导致 $\mathrm{T}_{\mathrm{H}} 2$ 细胞极化. 酰胺的邻位引入了两个氟原子(78)也可 优先诱导 $\mathrm{T}_{\mathrm{H}} 2$ 分化. 理论上, 作为吸电子基的氟原子应 增加 $\mathrm{N}-\mathrm{H}$ 键的极性, 加强氢键作用, 从而会使二元复 合物更加稳定, 优先诱导 $\mathrm{T}_{\mathrm{H}} 1$ 的分化. 但事实上, 可能 是由于氟原子的存在使 $\mathrm{C}-\mathrm{F}$ 与 $\mathrm{C}=\mathrm{O}$ 相互排斥, 结果导 致酰胺键轻微的扭转, 破坏了氢键体系, 降低二元复合 物的稳定性, 从而诱导了 $\mathrm{T}_{\mathrm{H}} 2$ 极化. 有意思的是, 用嫝 基取代酰胺的化合物 79 $^{[57]}$ 可诱导产生大量的 IFN- $\gamma$ ，从

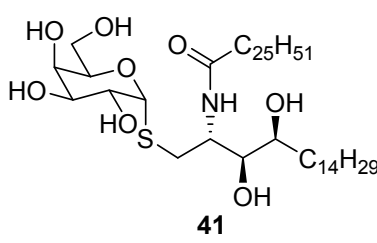

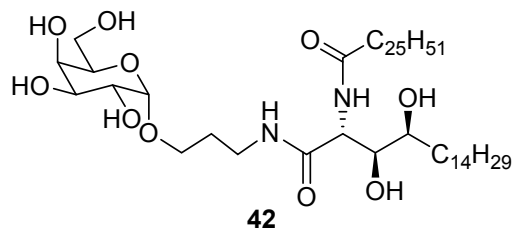

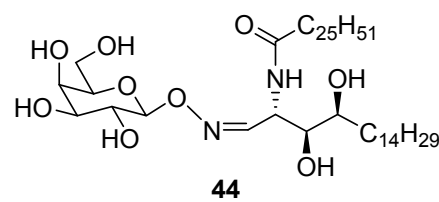

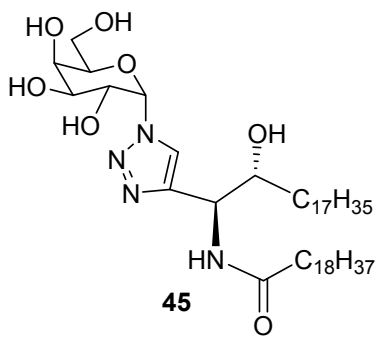

43

图 6 KRN7000 的 $S$-糖苷及其他衍生物

Figure 6 -glycoside and other analogs of KRN7000 


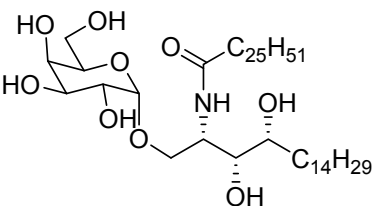

46

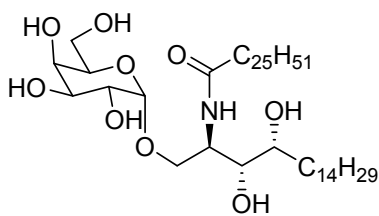

50

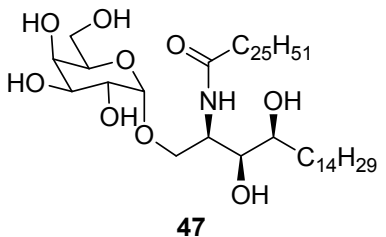

47

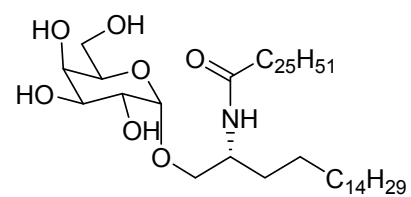

51

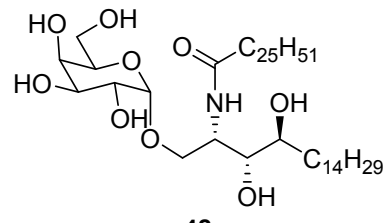

48

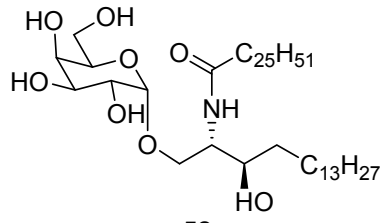

52

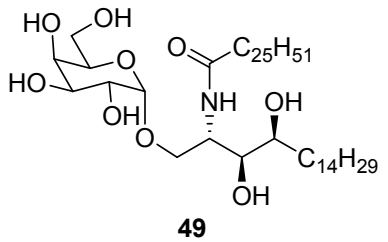

49

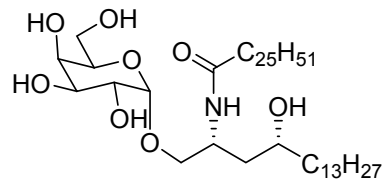

53

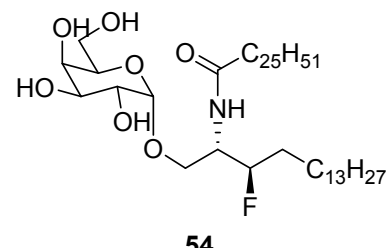

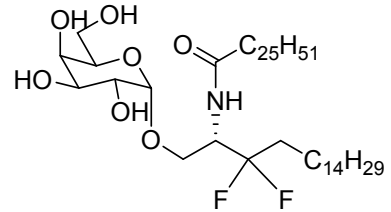

55

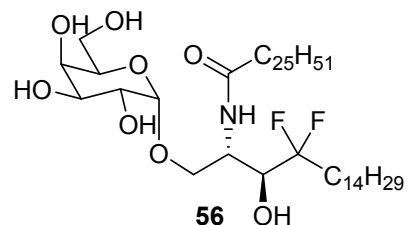

56

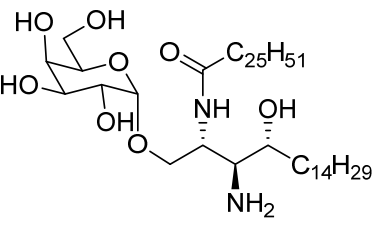

57

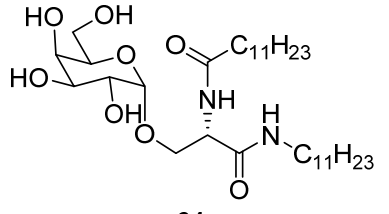

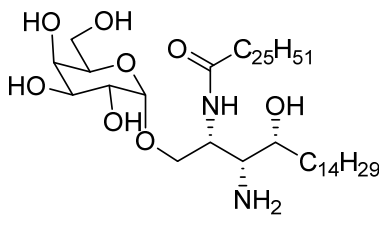

58

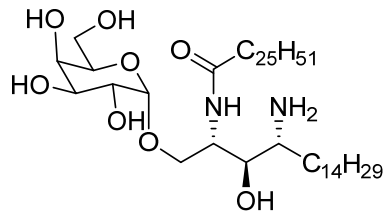

59

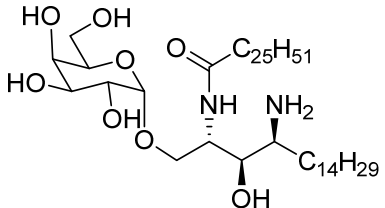

60

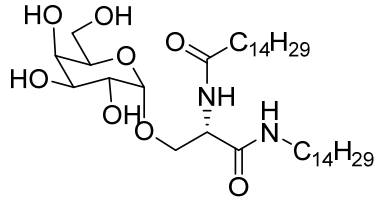

62

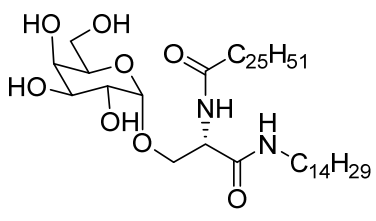

63

图 7 鞘氨醇结构改造的类似物 $46 \sim 63$

Figure 7 Modified analogs $46 \sim 63$ of the ceramide moiety

而导致 $\mathrm{T}_{\mathrm{H}} 1$ 细胞极化, 这可能与分子显著促进 IL- $12 \mathrm{p} 70$ 因子的生成有关. 而以硫嫝取代的化合物 $\mathbf{8 0}$, 由于硫原 子较大的体积, 导致活性缺失.

叶新山 ${ }^{[42] 、 S h i o z a k i ~}$ 等 $^{[58,59]}$ 用酯 $(81 \sim 88)$ 、醚 $(89 \sim$ 93)以及仲胺 $(94)$ 取代酰胺键, 除 $82 \sim 88$ 有轻微的 $\mathrm{T}_{\mathrm{H}} 2$ 免疫应答外, 其余衍生物均没有活性. 把 $\mathrm{N}$ 原子换成 $\mathrm{O}$ 原子, 将导致 OTAN 氢键体系中 $\mathrm{T}_{\mathrm{H}} \mathrm{r} 154$ 与 $\mathrm{N}-\mathrm{H}$ 氢键 的消失, 因此不能形成稳定的二元复合物可能是这些化 合物失活的主要原因.

其他酰胺的替代衍生物, 如 $\mathrm{N}$ 甲基化的化合物 $(95)^{[60]}$ 、氮杂环丁烷(96 和 97) 以及吡咯烷 $(98)^{[61]}$ 取代氨 基的化合物，免疫活性均不理想. 值得一提的是，化合 物 95 可以选择性地诱导 $\mathrm{T}_{\mathrm{H}} 2$ 分化, 而化合物 96 在较低 浓度时有剂量依赖性 $\mathrm{T}_{\mathrm{H}} 2$ 极化效应. 因此, 酰胺部分的 结构修饰大都表现出与 $\mathrm{T}_{\mathrm{H}} 2$ 极化相关的结果, 这将对研
究 KRN7000 及其衍生物用于自身免疫疾病治疗有很强 的指导意义.

\section{4 脂链 $\mathrm{D}$ 的结构修饰}

化合物 99(表 3)为脂链修饰衍生物中的第一个明显 对小鼠 NKT 细胞有应答的类似物, 并能优先诱导 $\mathrm{T}_{\mathrm{H}} 2$ 分化, 活性与 KRN7000 相当. 其余的化合物, 无论是缩 短鞘氨醇部分的脂链 $(100 \sim 105)$, 还是缩短酰胺连接的 脂链(106 和 107), 都和 99 一样可以优先诱导 $\mathrm{T}_{\mathrm{H}} 2$ 分 化 ${ }^{[62 ~ 64]}$, 但由于化合物活化 NKT 细胞的临界浓度要比 KRN7000 高得多, 因此相同的低浓度下, 不刺激 NKT 细胞应答. 化合物优先诱导 $\mathrm{T}_{\mathrm{H}} 2$ 分化的原因可能是任何 一条脂链上碳原子的减少都将会导致化合物和 CD1d 结 合力变弱或改变了结合方式，使解离速度加快. 而 IFN- $\gamma$ 的释放需要的刺激时间比 IL-4 的长, 因此 $\mathrm{T}_{\mathrm{H}} 2$ 分 化更占优势. 


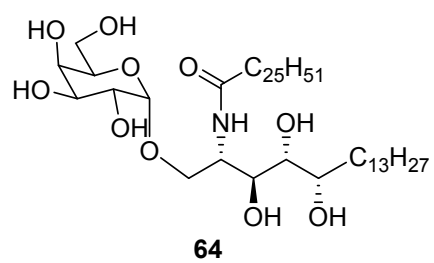

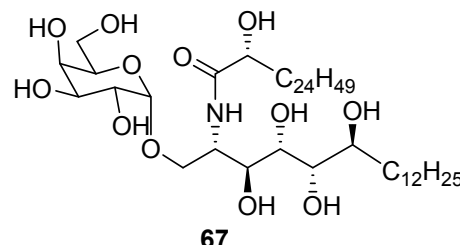

67

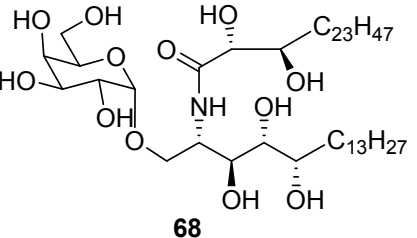

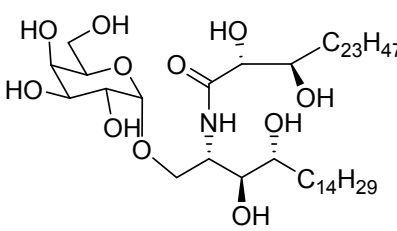

71

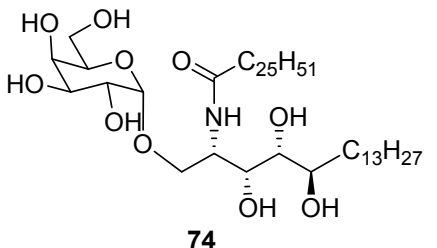

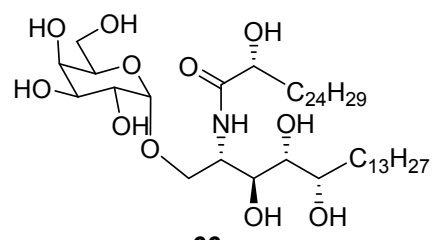

66

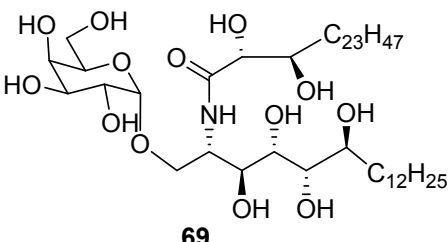

69

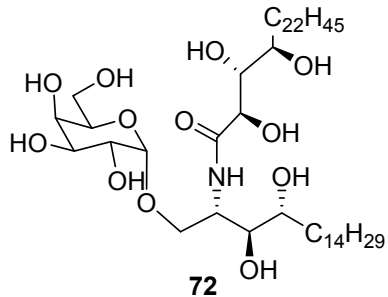

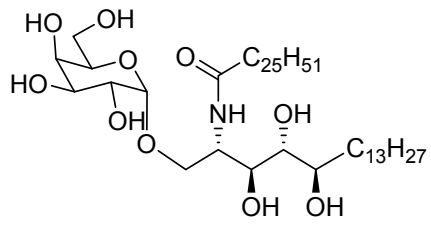

75

图 8 鞘氨醇结构改造的类似物 $64 \sim 75$

Figure 8 Modified analogs $64 \sim 75$ of the ceramide moiety

酰胺连接的脂肪链如果用 $Z$ 式不饱和链取代, 化合 物 108 111 同样可以增加 IL-4 的释放, 利于 $\mathrm{T}_{\mathrm{H}} 2$ 分化. 因此, 缩短直链的脂肪链或引入不饱和链可以使化合物 选择性的优先诱导 $\mathrm{T}_{\mathrm{H}} 2$ 分化 ${ }^{[65]}$. 而在其中之一的脂肪链 上引入芳基, 则往往得到相反结果. 如酰胺直接连接噻 吩环(112)或䒺环(113)的化合物可以优先诱导 $\mathrm{T}_{\mathrm{H}} 1$ 分化, 活性与 KRN7000 相当 ${ }^{[66]}$.

在鞘氨醇部分, 酯链以有苯基的 3 或 4 个亚甲基代 替, 所得化合物 114 116 ${ }^{[67]}$ 亦能优先诱导 $\mathrm{T}_{\mathrm{H}} 1$ 分化, 活 性略强于 KRN7000. 而酰胺脂肪链末端引入苯基, 化合 物对 $\mathrm{T}_{\mathrm{H}} 1$ 分化的诱导效果更加明显. 其连接臂的长度分 别为 5 个(117)、 7 个(118)或 10 个(119)亚甲基时, 诱导 $\mathrm{T}_{\mathrm{H}} 1$ 细胞分化效果最好, 且活性较 KRN7000 强 ${ }^{[26]}$. 但有 意思的是, Park 等 ${ }^{[68]}$ 基于此设计合成的鞘氨醇和酰胺脂 链末端都引入苯环的衍生物 $120 \sim 122$, 却都没有活性.

此外, Michieletti 等 ${ }^{[69]}$ 合成了鞘氨醇处连有醚氧链 的类似物 123 126(图 10), 活性均没有 KRN7000 的好, 表明鞘氨醇链上醚键的引入并不能提高化合物的活性.
而含有二唑杂环链的化合物 127, 其芳基可能通过与 CD1d 中 Tyr73、Phe77 和 Trp133 等残基的非共价结合, 却能更有效地刺激 IL-4 的表达, 优先诱导 $\mathrm{T}_{\mathrm{H}} 2$ 分化 ${ }^{[70]}$.

\section{5 其它结构修饰}

考虑到半乳糖基 6 位对化合物免疫活性影响不大， 在此处连接荧光基团作为探针较为合理，如化合物 128 131(活性与 KRN7000 相当) ${ }^{[71]}$. 此外, Sakai 等 ${ }^{[72 \sim 74]}$ 在酰胺链末引入了荧光基团 $(132 \sim 134$, 图 11), 其中化合物 133 能较好的活化 NKT 细胞, 但更重要的是 这些化合物可以用于研究 KRN7000 如何穿过细胞膜进 入细胞 ${ }^{[75]}$.

\section{3 结论}

在过去的十几年中，学者们试图寻找选择性更好的 KRN7000 类似物, 并对其免疫作用机理进行研究, 取得 了显著的成绩. 构效关系分析表明: 糖苷键的稳定性、 糖的 6 位疏水基团、较长的脂肪链及其末端芳香环的存 

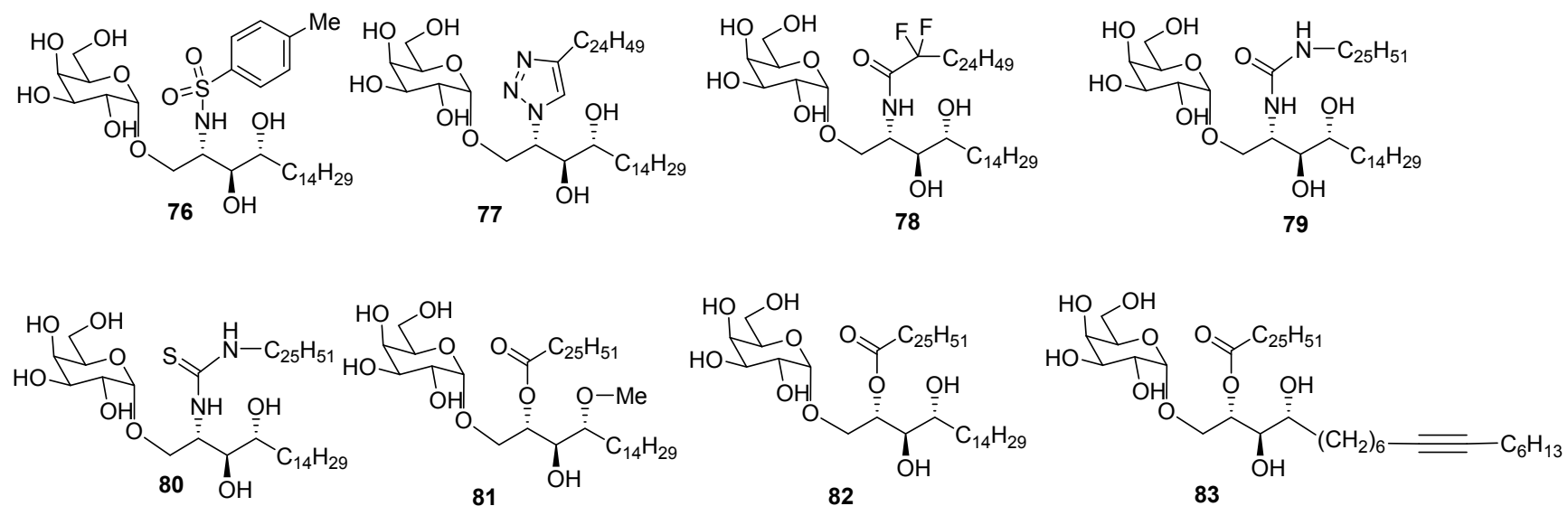

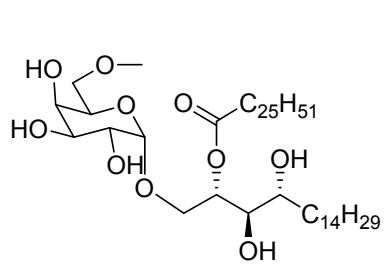

84

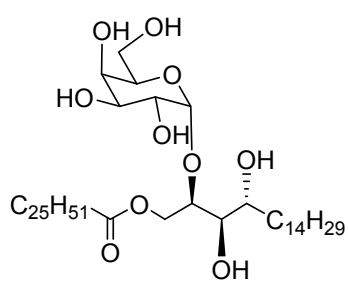

85

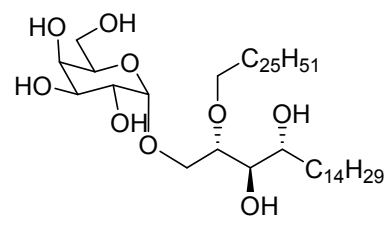

89

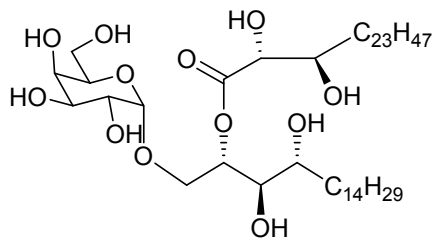

86

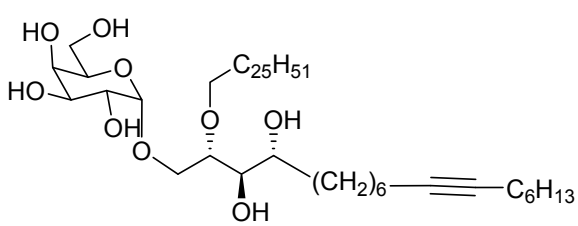

90
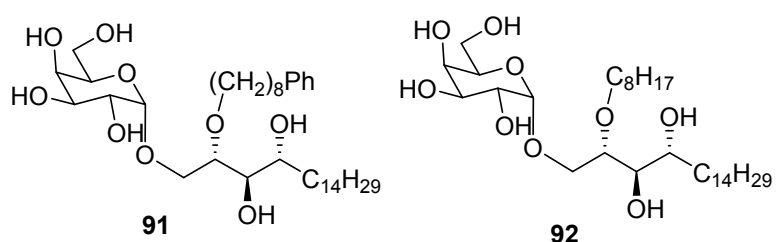

92

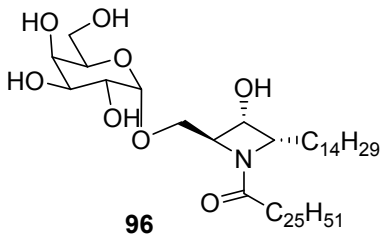

图 9 神经酰胺结构改造的类似物

Figure 9 Modified analogs of amide side chain

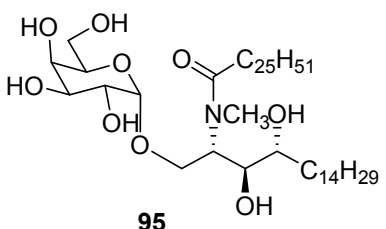

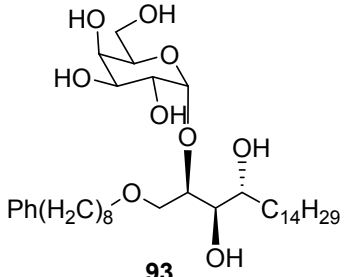

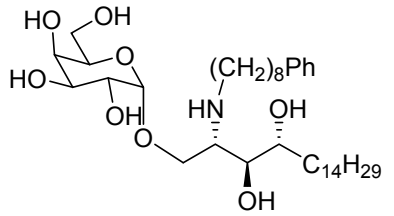

94
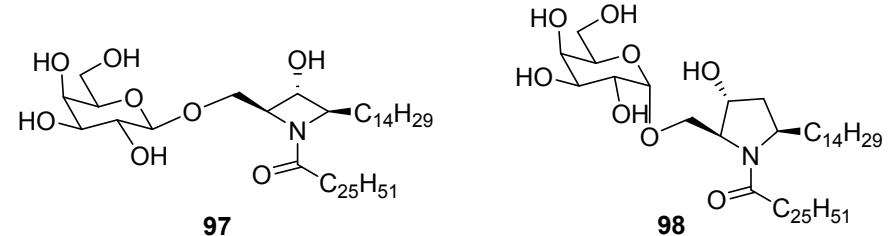

在等因素优先诱导 $\mathrm{T}_{\mathrm{H}} 1$ 的免疫反应; 而酰胺键的改变、 较短的脂肪链及其中的不饱和链的存在将会导致 $\mathrm{T}_{\mathrm{H}} 2$ 极化. 但是, KRN7000 如何影响细胞因子释放仍然是个 急需解决的重要问题? 同时, 在分子结构上, 为什么糖 基或神经酰胺上一点小小的变动将造成化合物与 $\mathrm{CD} 1 \mathrm{~d}$ 以及与 $\mathrm{TCR}$ 结合时的巨大差异, 并导致不同的细胞因
子的释放？因此，未来对 KRN7000进行结构修饰，仍应 从进一步增强 KRN7000 类似物和 $\mathrm{CD} 1 \mathrm{~d}$ 的有效结合，提 高二元/三元复合物的稳定性，并针对性的从期望得到 的细胞因子出发设计化合物，探讨更为深入的作用机 理. 
表 3 对脂链进行修饰的 KRN7000 的类似物

Table 3 Modification of the lipid chains

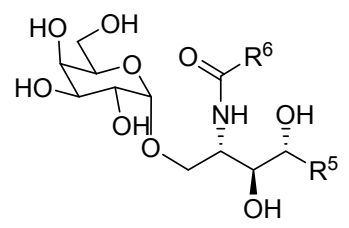

\begin{tabular}{|c|c|c|c|}
\hline Analogue & $\mathrm{R}^{5}$ & $\mathrm{R}^{6}$ & Activity $^{a}$ \\
\hline 99 & $\mathrm{C}_{5} \mathrm{H}_{11}$ & $\mathrm{C}_{23} \mathrm{H}_{47}$ & Similar $\mathrm{T}_{\mathrm{H}} 2^{\mathrm{b}}$ \\
\hline 100 & $\mathrm{C}_{2} \mathrm{H}_{5}$ & $\mathrm{C}_{23} \mathrm{H}_{47}$ & Slight increasing of $\mathrm{T}_{\mathrm{H}} 2$ \\
\hline 101 & $\mathrm{C}_{5} \mathrm{H}_{11}$ & $\mathrm{C}_{19} \mathrm{H}_{39}$ & Slight increasing of $\mathrm{T}_{\mathrm{H}} 2$ \\
\hline 102 & $\mathrm{C}_{5} \mathrm{H}_{11}$ & $\mathrm{C}_{25} \mathrm{H}_{51}$ & Slight increasing of $\mathrm{T}_{\mathrm{H}} 2$ \\
\hline 103 & $\mathrm{C}_{8} \mathrm{H}_{17}$ & $\mathrm{C}_{25} \mathrm{H}_{51}$ & Slight increasing of $\mathrm{T}_{\mathrm{H}} 2$ \\
\hline 104 & $\mathrm{C}_{11} \mathrm{H}_{23}$ & $\mathrm{C}_{25} \mathrm{H}_{51}$ & Slight increasing of $\mathrm{T}_{\mathrm{H}} 2$ \\
\hline 105 & $\mathrm{C}_{5} \mathrm{H}_{11}$ & $\mathrm{C}_{21} \mathrm{H}_{43} \sim \mathrm{C}_{24} \mathrm{H}_{49}$ & Similar $\mathrm{T}_{\mathrm{H}} 2$ \\
\hline 106 & $\mathrm{C}_{14} \mathrm{H}_{29}$ & $\mathrm{CH}_{3}$ & Slight increasing of $\mathrm{T}_{\mathrm{H}} 2$ \\
\hline 107 & $\mathrm{C}_{14} \mathrm{H}_{29}$ & $\mathrm{C}_{7} \mathrm{H}_{15}$ & Marked increasing of $\mathrm{T}_{\mathrm{H}} 2$ \\
\hline 108 & $\mathrm{C}_{14} \mathrm{H}_{29}$ & $\left(x_{9}=0\right.$ & Slight increasing of $\mathrm{T}_{\mathrm{H}} 2$ \\
\hline 109 & $\mathrm{C}_{14} \mathrm{H}_{29}$ & & Similar $\mathrm{T}_{\mathrm{H}} 2$ \\
\hline 110 & $\mathrm{C}_{14} \mathrm{H}_{29}$ & & Similar $\mathrm{T}_{\mathrm{H}} 2$ \\
\hline 111 & $\mathrm{C}_{5} \mathrm{H}_{11}$ & & Slight increasing of $\mathrm{T}_{\mathrm{H}} 2$ \\
\hline 112 & $\mathrm{C}_{14} \mathrm{H}_{29}$ & $\alpha$-Thienyl & Slight increasing of $\mathrm{T}_{\mathrm{H}} 1$ \\
\hline 113 & $\mathrm{C}_{14} \mathrm{H}_{29}$ & $\beta$-Naphthalenyl & Slight increasing of $\mathrm{T}_{\mathrm{H}} 1$ \\
\hline 114 & $\left(\mathrm{CH}_{2}\right)_{4} \mathrm{Ph}$ & $\mathrm{C}_{25} \mathrm{H}_{51}$ & Slight increasing of $\mathrm{T}_{\mathrm{H}} 1$ \\
\hline 115 & $\left(\mathrm{CH}_{2}\right)_{3} \mathrm{Ph}$ & $\mathrm{C}_{25} \mathrm{H}_{51}$ & Slight increasing of $\mathrm{T}_{\mathrm{H}} 1$ \\
\hline 116 & $\left(\mathrm{CH}_{2}\right)_{3} \mathrm{Ph}$ & $\mathrm{C}_{21} \mathrm{H}_{43}$ & Slight increasing of $\mathrm{T}_{\mathrm{H}} 1$ \\
\hline 117 & $\mathrm{C}_{14} \mathrm{H}_{29}$ & $\left(\mathrm{CH}_{2}\right)_{5} \mathrm{Ph}$ & Marked increasing of $\mathrm{T}_{\mathrm{H}} 1$ \\
\hline 118 & $\mathrm{C}_{14} \mathrm{H}_{29}$ & $\left(\mathrm{CH}_{2}\right)_{7} \mathrm{Ph}$ & Marked increasing of $T_{H} 1$ \\
\hline 119 & $\mathrm{C}_{14} \mathrm{H}_{29}$ & $\left(\mathrm{CH}_{2}\right)_{10} \mathrm{Ph}$ & Marked increasing of $T_{H} 1$ \\
\hline 120 & $\left(\mathrm{CH}_{2}\right)_{3} \mathrm{Ph}$ & $\left(\mathrm{CH}_{2}\right)_{5} \mathrm{Ph}$ & $-^{c}$ \\
\hline 121 & $\left(\mathrm{CH}_{2}\right)_{3} \mathrm{Ph}$ & $\left(\mathrm{CH}_{2}\right)_{7} \mathrm{Ph}$ & $-^{c}$ \\
\hline 122 & $\left(\mathrm{CH}_{2}\right)_{3} \mathrm{Ph}$ & $\left(\mathrm{CH}_{2}\right)_{9} \mathrm{Ph}$ & $-^{c}$ \\
\hline
\end{tabular}

${ }^{a} \mathrm{~T}_{\mathrm{H}} 1$ or $\mathrm{T}_{\mathrm{H}} 2$ stimulating activity compared to KRN7000; ${ }^{b}$ Weak NKT stimulation; ${ }^{c}$ No NKT cell activation.

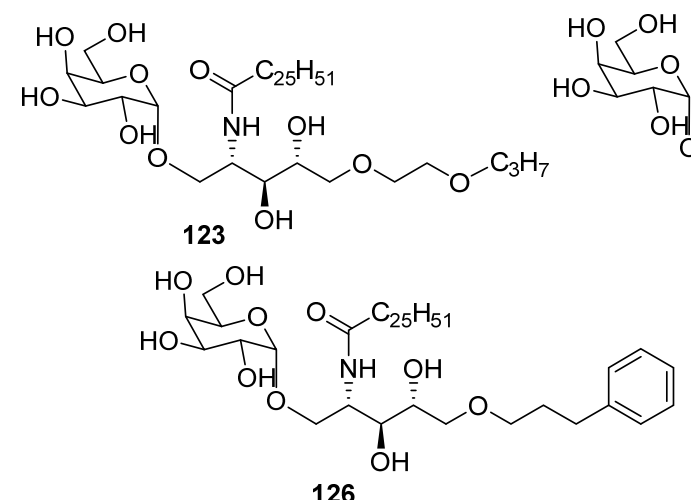

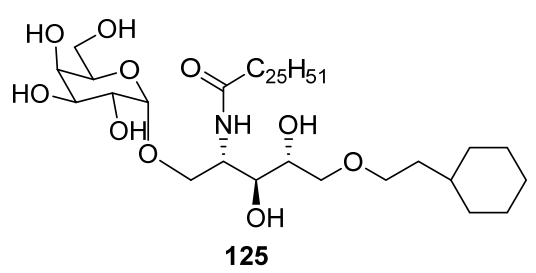

124

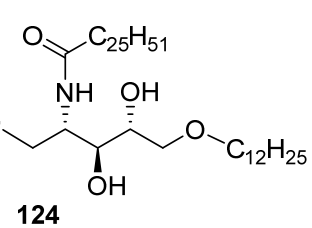

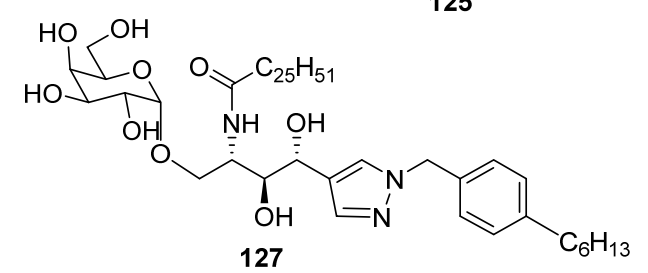

图 10 脂链结构改造的类似物

Figure 10 Modified analogs of the lipid side chain 


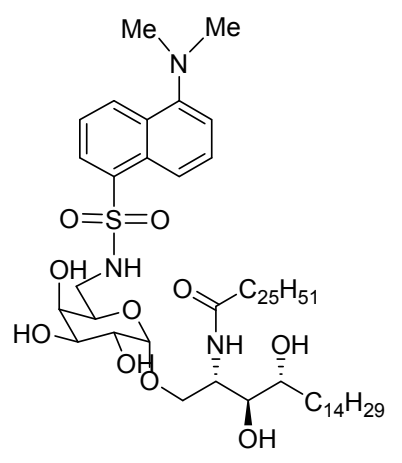

128

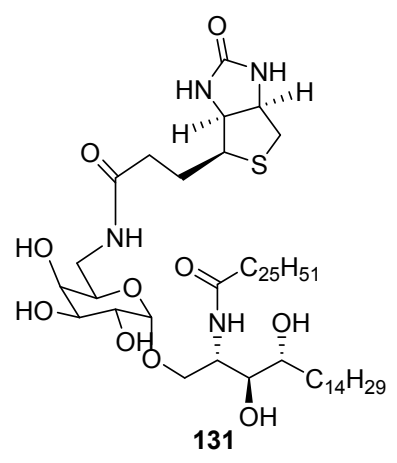<smiles>CCCCCCNc1ccc([N+](=O)[O-])c2nonc12</smiles>

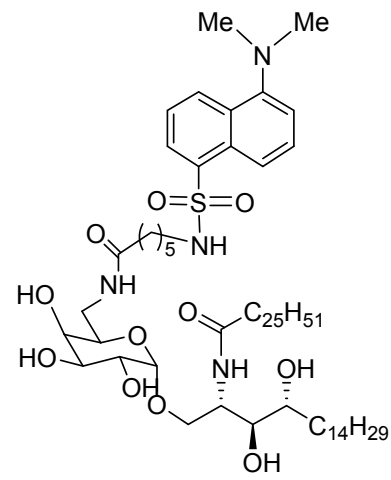

129

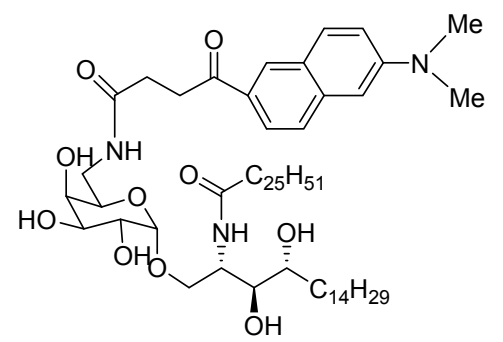

130

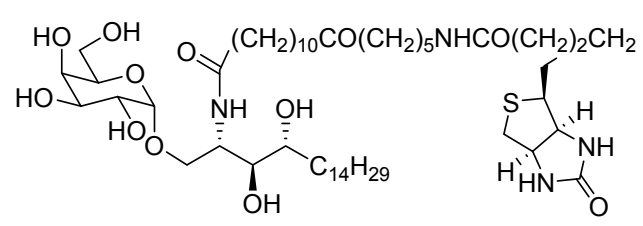

132

图 11 连有苂光基团的 KRN7000 衍生物

Figure 11 KRN7000 derivatives with the fluorescent group

\section{References}

[1] Natori, T.; Morita, M.; Akimoto, K.; Koezuka, Y. Tetrahedron 1994, 50, 2771.

[2] Uchida, T.; Horiguchi, S.; Tanaka, Y.; Yamamoto, H.; Kunii, N.; Motohashi, S.; Taniguchi, M.; Nakayama, T.; Okamoto, Y. Cancer Immunol. Immun. 2008, 57, 337.

[3] Chackerian, A.; Alt, J.; Perera, V.; Behar, S, M. Infect. Immun. 2002, 70, 6302.

[4] Hung, J.-T. Tsai, Y.-C.; Lin, W.-D.; Jan, J.-T.; Lin, K.-H.; Huang, J.-R.; Cheng, J.-Y.; Chen, M.-W.; Wong, C.-H.; Yu, A. L. Antiviral Res. 2014, 107, 110 .

[5] Kawakami, K.; Kinjo, Y,; Yara, S.; Koguchi, Y.; Uezu, K.; Nakayama, T.; Tanigushi, M.; Saito, A. Infect. Immun. 2001, 69, 213.

[6] Dieli, F.; Taniguchi, M.; Kronenberg, M.; Sidobre, S.; Ivanyi, J.; Fattorini, L.; Iona, E.; Orefici, G.; Leo, G. D.; Russo, D; Caccamo, N.; Sireci, G.; Sano, C. D.; Salerno, A. J. Immunol. 2003, 171, 1961.

[7] Singh, A. K.; Yang, J.-Q.; Parekh, V. V.; Wei, J.; Wang, C.-R.; Joyce, S.; Singh, R. R.; Kaer, L. V. Eur. J. Immunol. 2005, 35, 1143.

[8] Duarte, N.; Stenstrom, M.; Campino, S.; Bergman, M.-L.; Lundholm, M.; Holmberg, D.; Cardell, S. L. J. Immunol. 2004, 173,
3112.

[9] Kobayashi, E.; Motoki, K.; Yamaguchi, Y.; Uchida, T.; Fukushima, H.; Koezuka, Y. Bioorg. Med. Chem. Lett. 1996, 4, 615.

[10] Motohashi, S.; Nagato, K.; Kunii, N.; Yamamoto, H.; Yamasaki, K.; Okita, K.; Hanaoka, H.; Shimizu, N.; Suzuki, M.; Yoshino, I.; Taniguchi, M.; Fujisawa, T.; Nakayama, T. J. Immunol. 2009, 182, 2492.

[11] Singh, N.; Hong, S.; Scherer, D. C.; Serizawa, I.; Burdin, N.; Kronenberg, M.; Koezuka, Y.; Kaer, L. V. J. Immunol. 1999, 163, 2373.

[12] Yen, Y.-F.; Kulkarni, S. S; Chang, C.-W.; Luo, S. Y. Carbohydr. Res. 2013, 368, 35

[13] Franck, R. W.; Tsuji, M. Acc. Chem. Res. 2006, 39, 692.

[14] Zhang, L.; Ye. X. S. J. Chin. Pharm. Sci. 2008, 17, 263.

[15] Banchet-Cadeddu, A.; Hénon, E.; Dauchez, M.; Renault, J.-H.; Monneaux, F.; Haudrechy, A. Org. Biomol. Chem. 2011, 9, 3080.

[16] Nadas, J.; Li, C.; Wang, P. G. J. Chem. Inf. Model. 2009, 49, 410.

[17] Kawano, T.; Cui, J.; Koezuka, Y.; Toura, I.; Kaneko, Y.; Motoki, K.; Ueno, H.; Nakagawa, R.; Sato, H.; Kondo, E.; Koseki, H.; Taniguchi, M. Science 1997, 278, 1626.

[18] Raju, R.; Castillo, B. F.; Richardson, S. K.; Thakur, M.; Severins, R.; Kronenberg, M.; Howell, A. R. Bioorg. Med. Chem. Lett. 2009 , $19,4122$.

[19] Xing, G.-W.; Wu, D.; Poles, M. A.; Horowitz, A.; Tsuji, M.; Ho, D. D.; Wong, C.-H. Bioorg. Med. Chem. Lett. 2005, 13, 2907. 
[20] Zhou, D.; Mattner, J.; Cantu III, C.; Schrantz, N.; Yin, N.; Gao, Y.; Sagiv, Y.; Hudspeth, K.; Wu, Y.-P.; Yamashita, T.; Tenenberg, S.; Wang, D.; Proia, R. L.; Levery, S. B.; Savage, P. B.; Teyton, L.; Bendelac, A. Science 2004, 306, 1786.

[21] Veerapen, N.; Brigl, M.; S. Cerundolo, G, V.; Cox, L. R.; Brenner, M. B.; Besra, G. S. Bioorg. Med. Chem. Lett. 2009, 19, 4288.

[22] Jervis, P. J.; Graham, L. M.; Foster, E. L.; Cox, L. R.; Porcelli, S. A.; Besra, G. S. Bioorg. Med. Chem. Lett. 2012, 22, 4348.

[23] Tashiro, T.; Nakagawa, R.; Inoue, S.; Shiozaki, M.; Watarai, H.; Taniguchi, M.; Mori, K. Tetrahedron Lett. 2008, 49, 6827.

[24] Tashiro, T.; Nakagawa, R.; Shigeura, T.; Watarai, H.; Taniguchi, M.; Mori, K. Bioorg. Med. Chem. 2013, 21, 3066.

[25] Pauwels, N.; Aspeslagh, S.; Vanhoenacker, G.; Sandra, K.; Yu, E. D.; Zajonc, D. M.; Elewaut, D.; Linclau, B.; Van Calenbergh, S. Org. Biomol. Chem. 2011, 9, 8413.

[26] Chang, Y.-J.; Huang, J.-R.; Tsai, Y.-C.; Hung, J.-T.; Wu, D.; Fujio, M.; Wong, C.-H.; Yu, A. L. Proc. Natl. Acad. Sci. U. S. A. 2007, 104, 10299 .

[27] Ebensen, T.; Link, C.; Riese, P.; Schulze, K.; Morr, M.; Guzman, C. A. J. Immunol. 2007, 179, 2065.

[28] Tashiro, T.; Nakagawa, R.; Hirokawa, T.; Inoue, S.; Watarai, H.; Taniguchi, M.; Mori, K. Bioorg. Med. Chem. Lett. 2009, 17, 6360.

[29] Silk, J. D.; Salio, M.; Reddy, B. G.; Shepherd, D.; Gileadi, U.; Brown, J.; Masri, S. H.; Polzella, P.; Ritter, G.; Besra, G. S.; Jones, E. Y.; Schmidt, R. R.; Cerundolo, V. J. Immunol. 2008, 180, 6452.

[30] Diaz, G. Y. R.; Wojno, J.; Cox, L. R.; Besra, G. S. Tetrahedron: Asymmetry 2009, 20, 747.

[31] Tomiyama, H.; Yanagisawa, T.; Nimura, M.; Noda, A.; Tomiyama, T. JP 2001/354666, 2002 [Chem. Abstr. 2002, 136, 37901x; US 2002/0032158, 2002].

[32] Li, X.; Shiratsuchi, T.; Chen, G.; Dellabona, P.; Casorati, G.; Franck, R. W.; Tsuji, M. J. Immunol. 2009, 183, 4415.

[33] Altiti, A. S.; Mootoo, D. R. Org. Lett. 2014, 16, 1466.

[34] Liu, Z.; Byun, H. S.; Bittman, R. J. Org. Chem. 2011, 76, 8588.

[35] Liu, Z.; Byun, H. S.; Bittman, R. Org. Lett, 2010, 12, 2974.

[36] Toba, T.; Murata, K.; Yamamura, T.; Miyakeb, S.; Annoura, H. Tetrahedron Lett. 2005, 46, 5043.

[37] Pu, J.; Franck, R. W. Tetrahedron 2008, 64, 8618.

[38] Liu, Z.; Bittman, R. Org. Lett. 2012, 14, 620.

[39] Li, X.; Chen, G.; Garcia-Navarro, R.; Franck, R. W.; Tsuji, M. Immunology 2008, 127, 216.

[40] Lu, X; Song, L.; Metelitsa, L. S.; Bittman, R. ChemBioChem. 2006, $7,1750$.

[41] Blauvelt, M. L.; Khalili, M.; Jaung, W.; Paulsen, J.; Anderson, A. C.; Wilson, S. B.; Howell, A. R. Bioorg. Med. Chem. Lett. 2008, 18,6374 .

[42] Sun, M.; Wang, Y.; Ye, X.-S. Tetrahedron 2013, 69, 7438.

[43] Chen, W.; Xia, C.; Cai, L.; Wang, P. G. Bioorg. Med. Chem. Lett. 2010, 20, 3859.

[44] McDonagh, A. W.; Murphy, P. V. Tetrahedron 2014, 70, 3191.

[45] Park, J.-J.; Lee, J. H.; Ghosh, S. C.; Bricard, G.; Venkataswamy, M. M.; Porcelli, S. A.; Chung, S.-K. Bioorg. Med. Chem. Lett. 2008, $18,3906$.

[46] Lacone, V.; Hunault, J.; Pipelier, M.; Blot, V.; Lecoert, T.; Rocher, J.; Turcot-Dubois, A.-L.; Marionneau, S.; Douillard, J.-Y.; Clement, M.; Pendu, J. L.; Bonneville, M.; Micouin, L.; Dubreuil, D. J. Med. Chem. 2009, 52, 4960.

[47] Baek, D. J.; Seo, J.-H.; Lim, C.; Kim, J. H.; Chung, D. H.; Cho, W.-J.; Kang, C.-Y.; Kim, S. ACS Med. Chem. Lett. 2011, 2, 544.
[48] Hunault, J.; Diswall, M.; Frison, J.-C.; Blot, V.; Rocher, J.; Marionneau-Lambot, S.; Oullier, T.; Douillard, J.-Y.; Guillarme, S.; Saluzzo, C.; Dujardin, G.; Jacquemin, D.; Graton, J.; Questel, J.-Y. L.; Evain, M.; Lebreton, J.; Dubreuil, D.; Pendu, J. L.; Pipelier, M. J. Med. Chem. 2012, 55, 1227.

[49] Leung, L.; Tomassi, C.; Van Beneden, K.; Decruy, T.; Elewaut, D.; Elliott, T.; Al-Shamkhani, A.; Ottensmeier, C.; Calenbergh, S. V.; Werner, J.; Williams, T.; Linclau, B. Org. Lett. 2008, 10, 4433.

[50] Trappeniers, M.; Chofor, R.; Aspeslagh, S.; Li, Y.; Linclau, B.; Zajonc, D. M.; Elewaut, D.; Calenbergh, S. V. Org. Lett. 2010, 12, 2928.

[51] Fan, G.-T.; Pan, Y.-S.; Lu, K.-C.; Cheng, Y.-P.; Lin, W.-C.; Lin, S.; Lin, C.-H.; Wong, C.-H.; Fanga J.-M.; Lin, C.-C. Tetrahedron 2005, 61, 1855.

[52] Hung, L.-C.; Lin, C.-C.; Hung, S.-K.; Wu, B.-C.; Jan, M.-D.; Liou, S.-H. Fu, S.-L. Biochem. Pharmacol. 2007, 73, 1957.

[53] Shiozaki, M.; Tashiro, T.; Koshino, H.; Shigeura, T.; Watarai, H.; Taniguchi, M.; Mori, K. Carbohydr. Res. 2013, 370, 46.

[54] Shiozaki, M.; Tashiro, T.; Koshino, H.; Shigeura, T.; Watarai, H.; Taniguchi, M.; Mori, K. Bioorg. Med. Chem. 2014, 22, 827.

[55] Sawant, R. C.; Hung, J.-T.; Chuang, H.-L.; Lin, H.-S.; Chen, W.-S.; Yu, A. L.; Luo, S.-Y. Eur. J. Org. Chem. 2013, 7611.

[56] Lee, T.; Cho, M.; Ko, S.-Y.; Youn, H.-J.; Baek, D. J.; Cho, W.-J.; Kang, C.-Y.; Kim, S. J. Med. Chem. 2007, 50, 585.

[57] Tashiro, T.; Shigeura, T.; Watarai, H.; Taniguchi M.; Mori, K. Bioorg. Med. Chem. 2012, 20, 4540.

[58] Shiozaki, M.; Tashiro, T.; Koshino, H.; Nakagawa, R.; Inoue, S.; Shigeura, T.; Watarai, H.; Taniguchi, M.; Mori, K. Carbohydr. Res. 2010, 345, 1663.

[59] Shiozaki, M.; Tashiro, T.; Koshino, H.; Shigeura, T.; Watarai, H.; Taniguchi, M.; Mori, K. Tetrahedron 2013, 69, 9710

[60] Tashiro, T.; Shigeura, T.; Shiozaki, M.; Watarai, H.; Taniguchi, M.; Mori, K. Med. Chem. Commun. 2013, 4, 949.

[61] Fuhshuku, K.-I.; Hongo, N.; Tashiro, T.; Masuda, Y.; Nakagawa, R.; Seino, K.-I.; Taniguchi, M.; Mori, K. Bioorg. Med. Chem. 2008, 16,950 .

[62] Velmourougane, G.; Raju, R.; Bricard, G.; Im, J. S.; Besra, G. S.; Porcelli, S. A.; Howell, A. R. Bioorg. Med. Chem. Lett. 2009, 19, 3386.

[63] Toba, T.; Murata, K.; Nakanishi, K.; Takahashi, B.; Takemoto, N.; Akabane, M.; Nakatsuka, T.; Imajo, S.; Yamamura, T.; Miyake, S.; Annoura, H. Bioorg. Med. Chem. Lett. 2007, 17, 2781.

[64] Veerapen, N.; Reddington, F.; Salio, M.; Cerundolo, V.; Besra, G. S. Bioorg. Med. Chem. 2011, 19, 221.

[65] Yu, K. O. A.; Im, J. S.; Molano, A.; Dutronc, Y.; Illarionov, P. A.; Forestier, C.; Fujiwara, N.; Arias, I.; Miyake, S.; Yamamura, T.; Chang, Y.-T.; Besra, G. S.; Porcelli, S. A. Proc. Natl. Acad. Sci. U. S. A. 2005, 102, 3383.

[66] Fujio, M.; Wu, D.; Garcia-Navarro, R.; Ho, D. D.; Tsuji, M.; Wong, C.-H. J. Am. Chem. Soc. 2006, 128, 9022.

[67] Matto, P.; Modica, E.; Franchini, L.; Facciotti, F.; Mori, L.; Libero, G. D.; Lombardi, G.; Fallarini, S.; Panza, L.; Compostella, F.; Ronchetti, F. J. Org. Chem. 2007, 72, 7757.

[68] Park, J.-J.; Lee, J. H.; Seo, K.-C.; Bricard, G.; Venkataswamy, M. M.; Porcelli, S. A.; Chung, S.-K. Bioorg. Med. Chem. Lett. 2010, 20,814. 、

[69] Michieletti, M.; Bracci, A.; Compostella, F.; De Libero, G.; Mori, L.; Fallarini, S.; Lombardi, G.; Panza, L. J. Org. Chem. 2008, 73, 9192. 
[70] Kim, Y.; Kim, J.; Oh, K.; Lee, D.-S.; Park, S. B. ACS Med. Chem. Lett. 2012, 3, 151.

[71] Zhou, X.-T.; Forestier, C.; Goff, R. D.; Li, C.; Teyton, L.; Bendelac, A.; Savage, P. B. Org. Lett. 2002, 4, 1367.

[72] Sakai, T.; Naidenko, O. V.; Iijima, H.; Kronenberg, M.; Koezuka,
Y. J. Med. Chem. 1999, 42, 1836.

[73] Sakai, T.; Ehara, H.; Koezuka, Y. Org. Lett. 1999, 1, 359.

[74] Vo-Hoang, Y.; Micouin, L.; Ronet, C.; Gachelin, G.; Bonin, M. ChemBioChem 2003, 4, 27.

[75] Stocker, B. L.; Timmer, M. S. M. Carbohydr. Res. 2014, 389, 3.

(Qin, X.) 